\title{
Autofluorescence bronchoscopy to identify pre-cancerous bronchial lesions
}

\author{
L. Fuso1, G. Pagliari1, V. Boniello1, A. Trove'1, F .Varone1, \\ A. Longobardi1, S. Basso1, L. Trodella2
}

ABSTRACT: Autofluorescence bronchoscopy to identify pre-cancerous bronchial lesions. L. Fuso, G. Pagliari, V. Boniello, A. Trove', F.Varone, A. Longobardi, S. Basso, L. Trodella.

Background. This study aimed to assess the diagnostic yield of autofluorescence bronchoscopy (AFB) in the detection of pre-cancerous bronchial lesions in a non-selected sample of patients.

Methods. Both fiberoptic white-light bronchoscopy (WLB) and AFB using the Storz D-light system were performed on 166 consecutive patients. Biopsy specimens were taken in areas of the tracheobronchial tree judged as abnormal or suspicious at WLB and/or AFB. The bronchoscopic procedures were randomly performed by two operators.

Results. A total of 93 patients had a positive biopsy specimen: 80 for cancer and 13 for dysplasia. AFB was abnormal or suspicious in 85 of the 93 patients with a sensitivity of $91.4 \%$. Specificity was $50.7 \%$. In 16 patients with normal WLB examination, AFB identified abnormal or suspicious areas which had a positive biopsy. Thus AFB significantly improved sensitivity of WLB $(100 \%$ vs $82.8 \%$, respectively, $p<0.001$ ) in the entire sample of patients studied. Data was further analysed separately for patients with dysplasia and those with cancer. Indeed, 13 of 16 patients recognized only by AFB had a histological diagnosis of dysplasia. The remaining three patients had a diagnosis of cancer (small intraepithelial neoplastic lesions). Since no other patient with dysplasia was found, AFB had a sensitivity of $100 \%$ in diagnosing dysplasia. On the other hand, excluding the 13 patients with dysplasia, WLB had a high sensitivity in diagnosing cancer $(93.7 \%)$.

Conclusions. The AFB Storz system showed a high sensitivity. The increase in diagnostic yield of AFB in comparison with WLB was related to the power of AFB to identify pre-cancerous bronchial lesions so showing its usefulness in the early diagnosis of lung cancer. Monaldi Arch Chest Dis 2005; 63: 3, 124-128.

Keywords: Autofluorescence bronchoscopy, early lung cancer, pre-cancerous lesion, diagnostic yield.

${ }^{1}$ Respiratory Disease Department,

${ }^{2}$ Radiology Department, Catholic University, Rome, Italy.

Correspondence: Dr. Leonello Fuso; Fisiopatologia Respiratoria; Università Cattolica S. Cuore; Largo A. Gemelli 8, 00168 Roma,Italy; e-mail:leofuso@rm.unicatt.it

\section{Introduction}

Fluorescence bronchoscopy is recognised as a new technique playing an important role in the early detection of lung cancer [1]. Indications include known or suspected lung cancer by abnormal sputum cytology findings, inspection for synchronous tumours, surveillance following cancer resection, and primary screening among high-risk patients [2].

Autofluorescence Bronchoscopy (AFB) is a procedure in which premalignant and malignant tissue is distinguished by a change in colour from normal tissue without the need for fluorescenceenhancing drugs. The diagnostic yield of this method in patients with lung cancer has been reported in some papers: AFB showed better sensitivity for cancerous and pre-cancerous lesions than conventional White-Light Bronchoscopy (WLB) and the evaluation of the extent of cancer invasion was more accurate [3, 4]. These results were obtained using Laser-Induced Fluorescence Endoscopy (LIFE). Another system now currently used, the Storz D-light system, is less expensive and allows an immediate comparison between white-light and autofluorescence imaging [5]. This system has been recently tested in a multicenter study only for class III (severe dysplasia, carcinoma in situ, early invasive cancer) mucosal lesions and a significant increase in sensitivity in comparison with WLB has been found [6]. Moreover, it is showed that both LIFE and Storz systems yielded comparable results with only a difference in terms of examination time which was significantly shorter with the Storz system [7].

A potential limit of this technique is related to the interpretation of the AFB imaging, especially when the difference in colour between abnormal and normal areas is poor. In these cases, it is possible that the experience in interpreting the AFB imaging might play an important role.

The aim of this study was to verify the usefulness of AFB Storz system in identifying pre-cancerous bronchial lesions in a non-selected sample of consecutive patients who underwent bronchoscopy. 


\section{Methods}

\section{Patients}

One-hundred-sixty-six consecutive patients (120 males and 46 females), mean age $65 \pm 10$ years who underwent bronchoscopy were enrolled. An informed written consent was obtained from each patient and the study protocol was approved by the local ethical committee.

The indications to bronchoscopy were the following:

1. radiological abnormality at chest $\mathrm{x}$-ray or $\mathrm{CT}$ scan suspected for lung cancer (patients n. 73);

2. post-operative follow-up programme in patients who had previously undergone a complete surgical resection for non-small cell lung cancer (n. 39);

3. Chronic Obstructive Pulmonary Disease (COPD), especially in patients with a history of recent haemoptysis and/or worsening of respiratory symptoms (n. 54).

\section{Endoscopy}

The bronchoscopic procedure was performed under local anesthesia using the Storz D-light system (Karl Storz, Tuttlingen, Germany). This system consists of a fiberoptic bronchoscope and a Xenon lamp to produce a light with an emission spectrum from ultraviolet to blue corresponding to a wavelength from 380 to $460 \mathrm{~nm}$. The fluorescence response from the tissue is filtered and displayed on a monitor. Abnormal tissue is marked as a darkened reddish-brown area within the bluegreen coloured normal tissue [8]. A direct switching between the white-light and autofluorescence mode allows an immediate comparison between the imaging during the same procedure. The sequence of the examinations (WLB performed before or after AFB) was randomly defined for each patient before the procedure. The bronchoscopic procedures were randomly performed by two operators (G.P. and L.F.).

An area of the tracheobronchial tree was judged as abnormal at WLB in presence of a clear mucosal infiltration and/or a proliferative lesion; it was judged as suspicious if a slight thickening or irregularity of the mucosa was present. An abnormal AFB imaging included a well defined darkened red area, whereas a AFB imaging was considered suspicious in presence of patchy and heterogeneous defects in the green normal mucosal aspect. At least three consecutive biopsies were taken in each abnormal and suspicious area to obtain sufficient material for the histological examination. The results of the biopsy specimens were classified by a sin- gle pathologist unaware of bronchoscopic findings into the following categories: negative for cancerous or pre-cancerous lesions, dysplasia of moderate or severe grade, positive for cancer.

\section{Statistical analysis}

Suspicious and abnormal cases were considered together to calculate sensitivity, specificity, positive predictive value and negative predictive value of AFB. Differences in sensitivity and specificity between WLB considered alone and AFB in addition to WLB were analysed by a chi-square test.

\section{Results}

The results of AFB are reported in table 1. A total of 93 patients had a positive biopsy specimen: 80 for cancer and 13 for dysplasia. In 68 cases the AFB examination was abnormal and in 17 suspicious with a sensitivity of $91.4 \%$. Specificity was relatively low $(50.7 \%)$ and the negative predictive value $(82.2 \%)$ was better than the positive predictive value $(70.2 \%)$.

Table 2 shows the comparison between WLB and AFB. WLB did not identify 16 patients with a positive biopsy specimen taken in areas abnormal or suspicious only at AFB. Thus, the sensitivity significantly increased from $82.8 \%$ when WLB was considered alone to $100 \%$ when AFB

Table 1. - Results of autofluorescence bronchoscopy (AFB) compared to biopsy specimens

\begin{tabular}{lccc} 
& \multicolumn{2}{c}{ Biopsy specimen } & Total \\
\cline { 2 - 3 } & $\begin{array}{c}\text { Positive for cancer } \\
\text { or dysplasia }\end{array}$ & Negative & \\
\cline { 1 - 3 } AFB abnormal (n.) & 68 & 7 & 75 \\
AFB suspicious (n.) & 17 & 29 & 46 \\
AFB normal (n.) & 8 & 37 & 45 \\
\hline Total & 93 & 73 & 166 \\
\hline
\end{tabular}

Table 2. - Diagnostic yield of white light bronchoscopy (WLB) alone (upper panel) and together with autofluorescence bronchoscopy (AFB, lower panel)

\begin{tabular}{lccc}
\hline & \multicolumn{2}{c}{ Biopsy specimen } & Total \\
\cline { 2 - 3 } & $\begin{array}{c}\text { Positive for cancer } \\
\text { or dysplasia }\end{array}$ & Negative & \\
\hline WLB abnormal-suspicious (n.) & 77 & 16 & 93 \\
WLB normal (n.) & 16 & 57 & 73 \\
\hline Total & 93 & 73 & 166 \\
\hline WLB + AFB abnormal-suspicious (n.) & 93 & & 134 \\
WLB + AFB normal (n.) & 0 & 41 & 32 \\
\hline Total & 93 & 73 & 166 \\
\hline
\end{tabular}


was added to WLB $(\chi$-square $17.51, \mathrm{p}<0.001)$. On the other hand, specificity significantly decreased from $78.1 \%$ to $43.8 \%$ ( $\chi$-square 17.99 , $\mathrm{p}$ $<0.001)$. Figure 1 shows both WLB and AFB imaging of a positive case identified only by an abnormal AFB.

A further analysis was performed separately for patients with dysplasia and those with cancer. Indeed, 13 of 16 patients recognised only by AFB had a histological diagnosis of dysplasia. The remaining three patients had a diagnosis of cancer (small intraepithelial neoplastic lesions). Since no other patient with dysplasia associated with abnormal findings both at WLB and AFB was found, AFB had a sensitivity of $100 \%$ in diagnosing dysplasia. On the other hand, excluding the 13 patients with dysplasia, WLB was found to have a high sensitivity in diagnosing cancer $(93.7 \%)$.

\section{Discussion}

Several papers have shown the diagnostic usefulness of AFB in high-risk patients $[4,9,10]$, in staging of lung cancer [11, 12], in the follow-up of pre-cancerous lesions and after cancer resection [13-16].

Our main results were that sensitivity of AFB was high (table 1) and that the diagnostic yield significantly improved when AFB was used in addition to the conventional WLB examination (table 2 ). The increase in sensitivity was due to the identification by AFB of dysplasia which was not visible by WLB. This data confirms the usefulness of AFB in detecting pre-cancerous bronchial lesions and early cancer [6] and show that this technique is especially indicated in high-risk patients rather than in non-selected population [17]. On the other hand, AFB seems to be less useful in the diagnosis of cancer in more advanced phase. In this case, a high diagnostic yield could be obtained also by an accurate WLB examination alone.

Our study group had a relatively poor homogeneity because it included patients with several indications to bronchoscopy. However, the aim of the study was just to assess the diagnostic yield of AFB in non-selected consecutive patients. The great majority of them had either known or radiologically suspected lung cancer and, therefore, a high sensitivity of WLB in diagnosing cancer was found. All patients were at risk of developing lung cancer and this could explain the high percentage of pre-cancerous lesions identified and the increase in sensitivity obtained by AFB added to WLB examination.

Specificity of AFB derived from our data was relatively low in agreement with the known increase of false positive cases due to the non-specific abnormalities identified by AFB [18, 19]. As expected, we found that the negative predictive value was better $(82.2 \%)$ than the positive predictive value $(70.2 \%)$ due to a smaller number of false negative in comparison to false positive cases. However, the positive predictive value of AFB that derived from our data was satisfactory and comparable with other studies [3].

Actually, a comparison between our results and those obtained by other authors is only partially possible for differences in study design and study population. The sensitivity of AFB found by Kusunoki et al [4] was $83.7 \%$ but in this study a large number of biopsies were performed. In another paper, a very high sensitivity of AFB for diagnosis of cancer (97\%) was found in patients with lung cancer who received surgery [3]; however, thin sections from resected lung rather than biopsy specimens were considered as histological reference and a highly selected group was studied [3]. Moreover, a much lower sensitivity was found when diagnosis of dysplasia was considered separately [3].
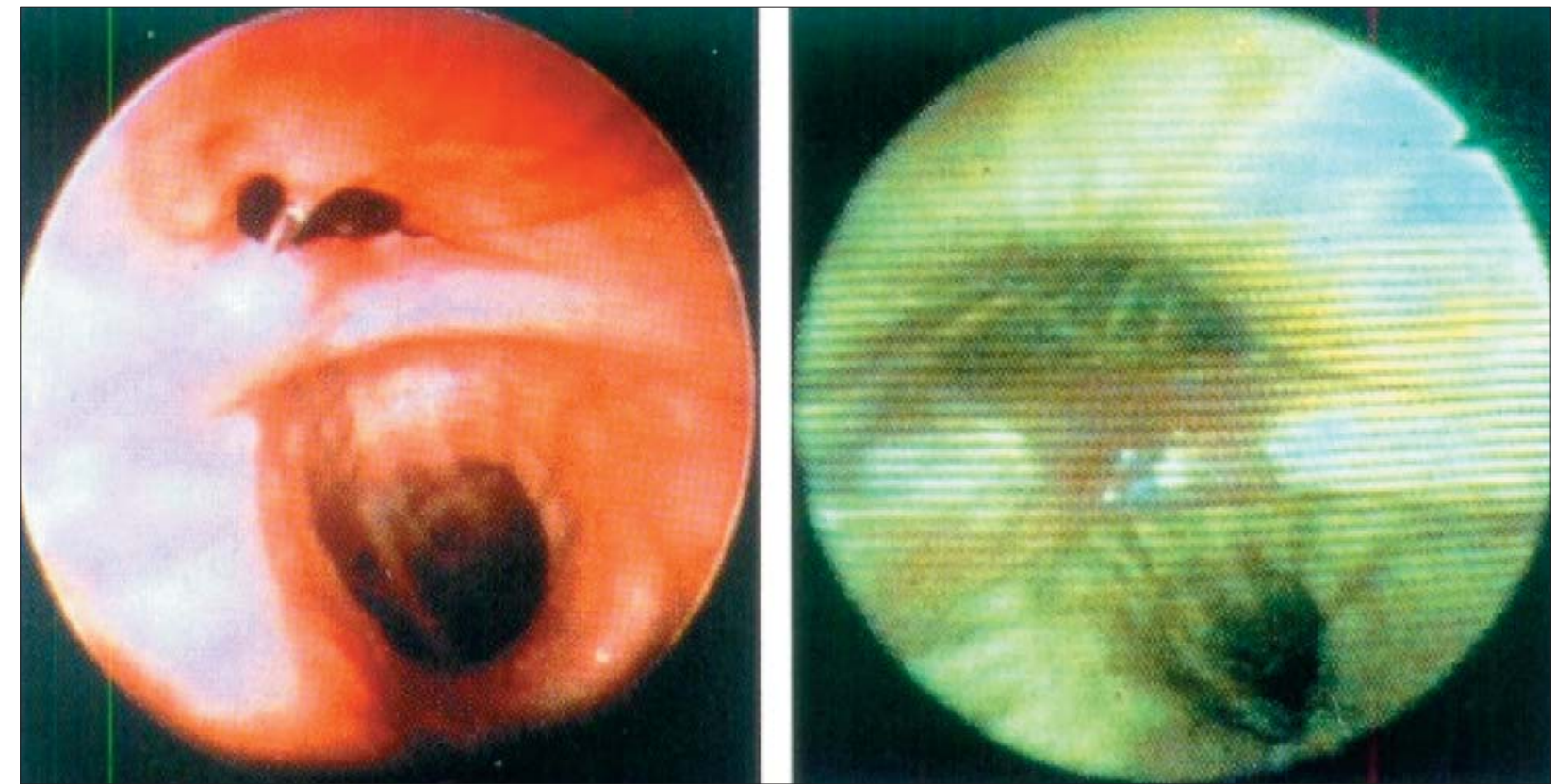

Fig. 1. - Normal white-light imaging (on the left) and abnormal autofluorescence imaging (on the right) of the same area (lingula carina): the histological specimen resulted positive for intraepithelial cancer. 
As noted above, our data showed that AFB was particularly useful in the detection of pre-cancerous lesions thereby increasing the diagnostic yield of the conventional WLB examination. The increase in sensitivity of AFB in comparison with WLB was reported in previous studies conducted using the LIFE system $[4,9,20]$. Lam et al studied a large population in a multicenter trial: the relative sensitivity of WLB and AFB used together versus WLB used alone increased especially for intraepithelial neoplastic lesions [9]. In another paper, the addition of autofluorescence resulted in a greater relative sensitivity over WLB alone in high-grade lesions (moderate to severe dysplasia and carcinoma in situ: relative sensitivity 2.4) and particularly in low-grade lesions (mild dysplasia: relative sensitivity 4.7) [10]. A significant improvement in sensitivity in comparison with WLB in diagnosing dysplasia or early cancer was also found using the AFB Storz system [6]. Our results were in agreement with these studies which demonstrated that the increase in sensitivity was related to the power of AFB to detect cancer in its pre-cancerous and/or early phase.

In our study, WLB was not able to identify any case of dysplasia and the sensitivity of AFB in diagnosing dysplasia was particularly high. Thus, the relative sensitivity of WLB and AFB used together versus WLB alone was quite different from that reported in literature in which it varies from 1.5 to $6[3,4,9,10,21]$. However, in the great majority of the previous studies the relative sensitivity was calculated in the whole sample of patients and not separately for cancer and for dysplasia. In our study the AFB sensitivity of $100 \%$ was related exclusively to patients with dysplasia. Moreover, the studies in which the relative sensitivity was separately calculated for cancer and for pre-neoplastic lesions included carcinoma in situ among pre-neoplastic lesions $[9,21]$. Patients with carcinoma in situ were not found in our sample. Final$1 y$, it is not usual that WLB can detect dysplasia. In fact, only in one study some cases of dysplasia (10 out of 92 low-grade lesions) were identified by WLB [10].

Limitations of our study deserve to be cited. The first is related to the relatively small number of biopsies examined. Another limitation is the lack of follow-up of the patients studied. This prevented us from verifying whether the early diagnosis of cancer or pre-cancerous lesions could influence the treatment and the prognosis of the patients. Moreover, it must be noted that sensitivity obtained in this study refers to a relative sensitivity because the true total number of lung cancer and pre-cancerous lesions was not verified after surgery or in a post-mortem study [22].

In conclusion, although partially influenced by some limitations, this study showed that the AFB Storz system is a sensitive tool in the diagnosis of lung cancer. The increase of the diagnostic yield in comparison with conventional bronchoscopy was due to the detection of pre-cancerous bronchial lesions by AFB. This characteristic could especially emphasise the role of AFB in early diagnosis and in screening of lung cancer in selected patients. A future aim could be to compare the results obtained by different bronchoscopists in a larger population to verify whether the experience in interpreting the AFB imaging may positively influence the diagnostic yield of this technique.

Acknowledgement: The authors wish to thank Mrs. L. Memmoli for her help in revising the manuscript.

\section{References}

1. Sutedja G. New techniques for early detection of lung cancer. Eur Respir J 2003; 21 (Suppl 39): 57s-66s.

2. Ernst A, Silvestri GA, Johnstone D, for the ACCP Interventional Chest/Diagnostic Procedures Network Steering Committee. Interventional pulmonary procedures. Guidelines from the American College of Chest Physicians. Chest 2003; 123: 1693-1717.

3. Ikeda N, Hiyoshi T, Kakihana M, et al. Histopathological evaluation of fluorescence bronchoscopy using resected lungs in cases of lung cancer. Lung Cancer 2003; 41: 303-309.

4. Kusunoki Y, Imamura F, Uda H, et al. Early detection of lung cancer with laser-induced fluorescence endoscopy and spectrofluorometry. Chest 2000; 118: 1776-1782.

5. Bolliger CT, Haussinger K. Early detection of lung cancer. Eur Respir Mon 1998; 9: 22-35.

6. Beamis JF, Ernst A, Mathur P, Yung R, Simoff M. A multi-center study comparing autofluorescence bronchoscopy to white light bronchoscopy. (abstr) Lung Cancer 2003; 41 (Suppl. 2): S49-S50.

7. Herth FJF, Ernst A, Becker HD. Autofluorescence bronchoscopy. A comparison of two systems (LIFE and D-Light). Respiration 2003; 70: 395-398.

8. Haussinger K, Pichler J, Stanzel F, et al. Autofluorescence bronchoscopy: the D-light system. In: Bolliger CT, Mathur PN, eds, Interventional bronchoscopy. Vol 30. Prog Respir Res. Basel: Karger, 2000: 243-252.

9. Lam S, Kennedy T, Unger M, et al. Localization of bronchial intraepithelial neoplastic lesions by fluorescence bronchoscopy. Chest 1998; 113: 696-702.

10. Moro-Sibilot D, Jeanmart M, Lantuejoul S, et al. Cigarette smoking, preinvasive bronchial lesions, and autofluorescence bronchoscopy. Chest 2002; 122: 19021908.

11. Sutedja TG, Codrington H, Risse EK, et al. Autofluorescence bronchoscopy improves staging of radiographically occult lung cancer and has an impact on therapeutic strategy. Chest 2001; 120: 1327-1332.

12. Pierard P, Vermylen P, Bosschaerts T, et al. Synchronous roentgenographically occult lung carcinoma in patients with resectable primary lung cancer. Chest 2000; 117: 779-785.

13. Pasic A, Vonk-Noordegraaf A, Risse EKJ, et al. Multiple suspicious lesions detected by autofluorescence bronchoscopy predict malignant development in the bronchial mucosa in high risk patients. Lung Cancer 2003; 41: 295-301.

14. Venmans BJW, van Boxem TJM, Smit EF, et al. Outcome of bronchial carcinoma in situ. Chest 2000; 117: 1572-1576.

15. Bota S, Auliac JB, Paris C, et al. Follow-up of bronchial pre-cancerous lesions and carcinoma in situ using fluorescence endoscopy. Am J Respir Crit Care Med 2001; 164: 1688-1693.

16. Weigel TL, Kosco PJ, Dacic S, et al. Postoperative flu- 
orescence bronchoscopic surveillance in non-small cell lung cancer patients. Ann Thorac Surg 2001; 71: 967970.

17. Kennedy TC, Lam S, Hirsch FR. Review of recent advances in fluorescence bronchoscopy in early localization of central airway lung cancer. The Oncologist 2001; 6: 257-262.

18. Kennedy TC, Miller Y, Prindiville S. Screening for lung cancer revisited and the role of sputum cytology and fluorescence bronchoscopy in a high-risk group. Chest 2000; 117: 72S-79S.

19. Van Zandwijk N. New methods for early diagnosis of lung cancer. Lung Cancer 2002; 38: S9-S11.
20. Sato M, Sakurada A, Sagawa M, et al. Diagnostic results before and after introduction of autofluorescence bronchoscopy in patients suspected of having lung cancer detected by sputum cytology in lung cancer mass screening. Lung Cancer 2001; 32: 247-253.

21. Vermylen P, Pierard P, Roufosse C, et al. Detection of bronchial preneoplastic lesions and early lung cancer with fluorescence bronchoscopy: a study about its ambulatory feasibility under local anaesthesis. Lung Cancer 1999; 25: 161-168.

22. Sutedja G, Venmans BJ, Smit EF, et al. Fluorescence bronchoscopy for early detection of lung cancer. A clinical perspective. Lung Cancer 2001; 34: 157-168.

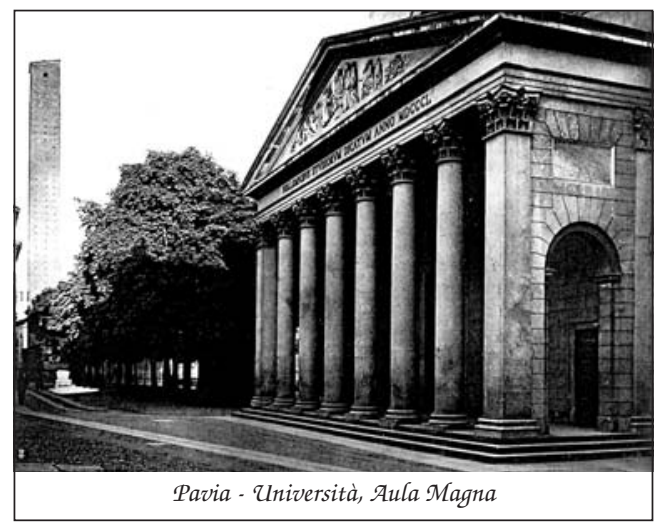

